\title{
SCATTERING OF WATER WAVES BY A SUBMERGED NEARLY CIRCULAR CYLINDER
}

\author{
B. N. MANDAL ${ }^{1}$ and SUDESHNA BANERJEA ${ }^{2}$
}

(Received 11 February 1992; revised July 1993)

\begin{abstract}
The problem of scattering of surface water waves by a horizontal circular cylinder totally submerged in deep water is well studied in the literature within the framework of linearised theory with the remarkable conclusion that a normally incident wave train experiences no reflection. However, if the cross-section of the cylinder is not circular then it experiences reflection in general. The present paper studies the case when the cylinder is not quite circular and derives expressions for reflection and transmission coefficients correct to order $\epsilon$, where $\epsilon$ is a measure of small departure of the cylinder cross-section from circularity. A simplified perturbation analysis is employed to derive two independent boundary value problems (BVP) up to first order in $\epsilon$. The first BVP corresponds to the problem of water wave scattering by a submerged circular cylinder. The reflection coefficient up to first order and the first order correction to the transmission coefficient arise in the second BVP in a natural way and are obtained by a suitable use of Green's integral theorem without solving the second BVP. Assuming a Fourier expansion of the shape function, these are evaluated approximately. It is noticed that for some particular shapes of the cylinder, these vanish. Also, the numerical results for the transmission coefficients up to first order for a nearly circular cylinder for which the reflection coefficients up to first order vanish, are given in tabular form. It is observed that for many other smooth cylinders, the result for a circular cylinder that the reflection coefficient vanishes, is also approximately valid.
\end{abstract}

\section{Introduction}

Assuming linear theory, the two-dimensional problems of water wave scattering by an obstacle present in water admit exact solutions only for a limited few cases, for example, when the obstacle is in the form of a thin vertical barrier. However, an integral equation formulation is always possible for obstacles of any geometrical shape by a judicious use of Green's integral theorem. The resulting integral equation can be solved approximately by analytical methods in some cases and numerically in most

\footnotetext{
${ }^{1}$ Physics and Appl. Math. Unit, Indian Statistical Institute, 203, B. T. Road, Calcutta 700 035, India.

${ }^{2}$ Department of Mathematics, Jadaupur University, Calcutta 700 032, India.

(C) Australian Mathematical Society, 1994, Serial-fee code 0334-2700/94
} 
cases. There are other procedures available in the literature for tackling these problems which essentially depend on the geometry of the obstacle. For example, when the obstacle is in the form of an infinitely long submerged cylinder with horizontal axis, Dean [1] used the technique of complex variables and Ursell [9] used the method of multipoles wherein a system of multipoles are placed at the centre of the cylinder and the strength of the multipoles satisfy an infinite linear system which always possesses an unique solution. Ogilvie [8] considered some more general situations in which Ursell's case was included, and used a similar technique to solve it. Levine [4] later used an integral equation formulation as mentioned above and considered obliquely incident wave trains also. They obtained the remarkable conclusion that a normally incident wave train experiences no reflection by the circular cylinder. However if the obstacle is not in the form of a circular cylinder, the wave train in general experiences reflection and in principle can be tackled by an integral equation formulation. For an obstacle in the form of a nearly circular cylinder, a simplified perturbation analysis can be utilized to handle the problem. A somewhat similar idea of perturbation ànalysis was used in some recent works involving scattering or radiation of water waves by nearly vertical barrier or plates (see Mandal and Chakrabarti [6], Mandal and Kundu [7] and Mandal and Banerjea [5]).

In the present paper, we consider water-wave scattering by a submerged nearly circular cylinder whose axis is horizontal. The aforesaid simplified perturbation technique is employed to reduce the problem up to first order to two independent BVPs. The first BVP corresponds to the problem of scattering of a normally incident wave train by a submerged circular cylinder, and it is studied by Dean [1], Ursell [9], Ogilvie [8], Levine [4] by various mathematical methods as mentioned earlier.

Using a tailored version of Green's integral theorem, based on an idea of Evans [2], the reflection coefficient up to first order and first order correction to transmission coefficient are obtained from the second BVP without actually solving it. Approximate expressions for these quantities are obtained by assuming a general Fourier expansion of the shape function representing the cross-section of the cylinder. It is found that for some special shapes, these quantities vanish. Also, when only a three-term Fourier expansion of the shape function is taken, the reflection coefficient up to first order is found to vanish. Thus if a nearly circular cylinder has sufficient continuity, then the Fourier coefficients in the Fourier expansion of the shape function will decay quickly, and a three-term series might be an excellent approximation to many fairly smooth cylinders. This implies that for many other smooth cylinders, the result that the reflection coefficient vanishes for a circular cylinder, also holds good approximately. 


\section{Formulation of the problem}

A rectangular cartesian coordinate system is used in which the $y$-axis is directed vertically downwards, and the origin lies on the axis of an infinitely long nearly circular cylinder submerged in deep water with its generators parallel to the $z$-axis and $f$ is the depth of its axis below the mean free surface. It is represented by $r=a\{1+\epsilon c(\theta)\},(0 \leq \theta \leq 2 \pi$ with $x=r \sin \theta, y=r \cos \theta)$ where $\epsilon$ is a small nondimensional parameter and is a measure of small departure of the cylinder crosssection from circularity, and $c(\theta)$ is a smooth function of $\theta$. A time-harmonic surface water wave train is normally incident on the cylinder from negative $x$-direction. The problem is two-dimensional and is independent of $z$. The motion is assumed to be irrotational and can be described by a velocity potential. Let the incident wave field be represented by $\operatorname{Re}\left\{\phi^{\text {inc }}(x, y) e^{-i \sigma t}\right\}$ where $\phi^{\text {inc }}(x, y)$ is given below and $\sigma$ is the angular frequency. Assuming linear theory, the motion in the fluid can be described by the velocity potential $\operatorname{Re}\left\{\phi(x, y) e^{-i \sigma t}\right\}$, where $\phi$ satisfies

$$
\begin{aligned}
\nabla^{2} \phi=0 & \text { in the fluid region, } \\
K \phi+\phi_{y}=0 & \text { on } y=-f
\end{aligned}
$$

where $K=\sigma^{2} / g, g$ being the gravity;

$$
\frac{\partial \phi}{\partial n}=0 \quad \text { on } r=a\{1+\epsilon c(\theta)\}, \quad 0 \leq \theta \leq 2 \pi
$$

where $n$ is normal to the surface of the cylinder;

$$
\begin{gathered}
\nabla \phi \rightarrow 0 \quad \text { as } y \rightarrow \infty \\
\phi \rightarrow \begin{cases}\phi^{\mathrm{inc}}(x, y)+R \phi^{\mathrm{inc}}(-x, y) & \text { as } x \rightarrow-\infty, \\
T \phi^{\mathrm{inc}}(x, y) & \text { as } x \rightarrow \infty,\end{cases}
\end{gathered}
$$

where $\phi^{\text {inc }}(x, y)=e^{-K(y+f)+i K x}$, and $R$ and $T$ denote reflection and transmission coefficients respectively and are to be determined.

\section{Method of solution}

The condition (2.3) can be approximated to the first order of $\epsilon$ as (see Appendix)

$$
\frac{\partial \phi}{\partial r}-\epsilon\left[c(\theta) \frac{\partial \phi}{\partial r}+\frac{1}{a} \frac{d}{d \theta}\left\{c(\theta) \frac{\partial \phi}{\partial \theta}\right\}\right]+\mathrm{O}\left(\epsilon^{2}\right)=0 \quad \text { on } r=a
$$


This form of boundary condition suggests that we may assume the following perturbation expansion for $\phi(x, y), R$ and $T$ in terms of $\epsilon$ as

$$
\left.\begin{array}{rl}
\phi(x, y ; \epsilon) & =\phi_{0}(x, y)+\epsilon \phi_{1}(x, y)+\mathrm{O}\left(\epsilon^{2}\right), \\
R & =R_{0}+\epsilon R_{1}+\mathrm{O}\left(\epsilon^{2}\right), \\
T & =T_{0}+\epsilon T_{1}+\mathrm{O}\left(\epsilon^{2}\right) .
\end{array}\right\}
$$

Substituting (3.2) in (3.1) and following the usual procedure for straightforward perturbation technique, we find $\phi_{0}(x, y)$ and $\phi_{1}(x, y)$ to be the solutions of the following two independent BVPs.

BVP I. $\phi_{0}(x, y)$ satisfies

$$
\begin{aligned}
& \nabla^{2} \phi_{0}=0 \quad \text { in } y>-f, r>a, \\
& K \phi_{0}+\frac{\partial \phi_{0}}{\partial y}=0 \quad \text { on } y=-f \text {, } \\
& \frac{\partial \phi_{0}}{\partial r}=0 \quad \text { on } r=a \text {, } \\
& \nabla \phi_{0} \rightarrow 0 \quad \text { as } y \rightarrow \infty \text {, } \\
& \phi_{0} \rightarrow \begin{cases}\phi^{\mathrm{inc}}(x, y)+R_{0} \phi^{\mathrm{inc}}(-x, y) & \text { as } x \rightarrow-\infty, \\
T_{0} \phi^{\mathrm{inc}}(x, y) & \text { as } x \rightarrow \infty .\end{cases}
\end{aligned}
$$

BVP II. $\quad \phi_{1}(x, y)$ satisfies

$$
\begin{aligned}
\nabla^{2} \phi_{1} & =0 \quad \text { in } y>-f, r>a, \\
K \phi_{1}+\frac{\partial \phi_{1}}{\partial y} & =0 \quad \text { on } y=-f, \\
\frac{\partial \phi_{1}}{\partial r} & =h(\theta) \quad \text { on } r=a, \\
\text { where } \quad h(\theta) & =\frac{1}{a} \frac{d}{d \theta}\left[c(\theta) \frac{\partial \phi_{0}}{\partial \theta}(\theta)\right] \\
\text { with } \quad \phi_{0}(\theta) & =\phi_{0}(a \sin \theta, a \cos \theta), \\
\nabla \phi_{1} & \rightarrow 0 \quad \text { as } y \rightarrow \infty, \\
\phi_{1} & \rightarrow \begin{cases}R_{1} \phi^{\text {inc }}(-x, y) & \text { as } x \rightarrow-\infty, \\
T_{1} \phi^{\text {inc }}(x, y) & \text { as } x \rightarrow \infty .\end{cases}
\end{aligned}
$$

We note that the BVP I corresponds to the two-dimensional problem of water wave scattering by an infinitely long horizontal circular cylinder submerged in deep water. As stated in the introduction this problem is well studied in the literature. In the 
condition (3.5) of BVP II we require the knowledge of $\phi_{0}(\theta)$. It is given by [4]

$$
\phi_{0}(\theta)=\phi^{\mathrm{inc}}(\theta)+\sum_{n=0}^{\infty} a_{n} e^{-i n \theta}, \quad 0 \leq \theta \leq 2 \pi .
$$

In (3.9) $a_{n}$ 's $(n \geq 1)$ satisfy the linear system

$$
\begin{aligned}
\sum_{m=1}^{\infty} a_{m} K_{m n}=(-1)^{n} \pi \frac{(K a)^{n}}{(n-1) !}, \quad n \geq 1, \\
K_{m n}=(-1)^{m+n} \frac{\pi}{2}\left(\frac{a}{2 f}\right)^{m+n} \frac{(m+n-1) !}{(m-1) !(n-1) !} \\
\quad-\frac{\pi(K a)^{m+n}}{(m-1) !(n-1) !}\left[\frac{d^{m+n}}{d \mu^{m+n}} F(\mu)\right]_{\mu=2 K f}, \quad m \neq n \\
K_{0} n=0 \quad \text { for all } n \geq 1, \\
K_{n n}=\frac{n \pi}{2}+\frac{\pi}{2}\left(\frac{a}{2 f}\right)^{2 n} \frac{(2 n-1) !}{\{(n-1) !\}^{2}}-\frac{\pi(K a)^{2 n}}{\{(n-1) !\}^{2}}\left[\frac{d^{2 n}}{d \mu^{2 n}} F(\mu)\right]_{\mu=2 K f},
\end{aligned}
$$

with

$$
F(\mu)=\int_{0}^{\infty} \frac{e^{-\mu z}}{z-1} d z
$$

where the path of integration is indented below the pole at $z=1$. It is also known that

$$
R_{0}=0
$$

while

$$
T_{0}=1+2 \pi i e^{-2 K f} \sum_{n=1}^{\infty} a_{n} \frac{(-1)^{n}(K a)^{n}}{(n-1) !}
$$

and the results are independent of $a_{0}$. The $a_{n}$ 's in (3.13) are determined approximately by truncating the linear system (3.10).

In order to obtain $R_{1}$, we utilize Evans' [2] idea of the application of Green's integral theorem to the harmonic functions $\phi_{1}(x, y)$ and $\phi_{0}(x, y)$ in the region bounded by the lines $y=-f,-X \leq x \leq X ; x=X,-f \leq y \leq Y ; y=Y,-X \leq x \leq X$; $x=-X,-f \leq y \leq Y$ and the circle $r=a$, and ultimately make $X, Y \rightarrow \infty$ so that we find

$$
R_{1}=i a \int_{0}^{2 \pi} \phi_{0}(\theta) h(\theta) d \theta
$$

After integrating by parts and noting that $\theta=0,2 \pi$ correspond to the same point on the circle, we see that

$$
R_{1}=-i \int_{0}^{2 \pi}\left[\frac{\partial \phi_{0}}{\partial \theta}(\theta)\right]^{2} c(\theta) d \theta
$$


Similarly to obtain $T_{1}$ we apply Green's integral theorem to the functions $\phi_{1}(x, y)$ and $\phi_{0}(-x, y)$ in the same region and obtain

$$
\begin{aligned}
T_{1} & =i a \int_{0}^{2 \pi} \phi_{0}(-\theta) h(\theta) d \theta \\
& =-i \int_{0}^{2 \pi} \frac{\partial \phi_{0}}{\partial \theta}(\theta) \frac{\partial \phi_{0}}{\partial \theta}(-\theta) c(\theta) d \theta .
\end{aligned}
$$

With the knowledge of $\phi_{0}(\theta)$ given in (3.9), we find

$$
\begin{aligned}
R_{1}=i \int_{0}^{2 \pi} c(\theta) & {\left[(K a)^{2} \exp (-2 K f) \exp \{-2 i \theta-2 K a \exp (-i \theta)\}\right.} \\
& -2 K a \exp (-K f) \sum_{n=1}^{\infty} n a_{n} \exp \{-i(n+1) \theta\} \exp \{-i \theta-K a \exp (-i \theta)\} \\
& \left.+\sum_{m=1}^{\infty} \sum_{n=1}^{\infty} m n a_{m} a_{n} \exp \{-i(m+n) \theta\}\right] d \theta
\end{aligned}
$$

and

$$
\begin{aligned}
T_{1}=i \int_{0}^{2 \pi} c(\theta)[ & (K a)^{2} \exp (-2 K f-2 K a \cos \theta) \\
& -2 K a \exp (-K f) \sum_{n=1}^{\infty} n a_{n} \exp (-K a \cos \theta) \cos \{K a \sin \theta+(n-1) \theta\} \\
& \left.+\sum_{m=1}^{\infty} \sum_{n=1}^{\infty} m n a_{m} a_{n} \exp \{-i(m-n) \theta\}\right] d \theta
\end{aligned}
$$

We now assume a general Fourier expansion of $c(\theta)$ as

$$
c(\theta)=\frac{t_{0}}{2}+\sum_{p=1}^{\infty}\left[s_{p} \sin p \theta+t_{p} \cos p \theta\right], \quad 0 \leq \theta \leq 2 \pi .
$$

Substituting (3.18) in (3.16) and (3.17) and evaluating the various integrals (see [9], page 488) we find $R_{1}$ and $T_{1}$ as

$$
\begin{aligned}
R_{1}=\pi \sum_{p=2}^{\infty}\left(s_{p}+i t_{p}\right)\left[\frac{(2 K a)^{p}}{2(p-2) !} \exp (\right. & -2 K f)+\sum_{n=1}^{p-1} n(p-n) a_{n} a_{p-n} \\
& \left.-2 \exp (-K f) \sum_{n=1}^{p-1} \frac{n a_{n}(K a)^{p-n}}{(p-n-1) !}\right]
\end{aligned}
$$


and

$$
\begin{aligned}
T_{1}=2 \pi i \sum_{p=1}^{\infty} t_{p}\left[(K a)^{2} \exp (-2 K f) I_{p}(2 K a)\right. \\
\left.\quad-\exp (-K f)\left\{\sum_{n=p+1}^{\infty} \frac{(K a)^{n-p}}{(n-p-1) !} n a_{n}+\sum_{n=1}^{\infty} n \frac{a_{n}(K a)^{n+p}}{(n+p-1) !}\right\}\right] \\
+\pi i\left[\sum_{n=1}^{\infty} \sum_{m=n+1}^{\infty} 2 m n a_{m} a_{n} t_{m-n}+t_{0} \sum_{n=1}^{\infty} n^{2} a_{n}^{2}\right. \\
\left.+i \exp (-2 K f)(K a)^{2} I_{0}(2 K a) t_{0}-2 \exp (-K f) t_{0} \sum_{n=1}^{\infty} \frac{(K a)^{n} n a_{n}}{(n-1) !}\right]
\end{aligned}
$$

where $I_{p}(z)$ denotes the modified Bessel function of first kind.

The constants, $a_{n}$, are obtained from the solution of the linear system (3.10) by truncation and the $t_{p}$ and $s_{p}$ 's are known once the shape function $c(\theta)$ is given. We note that $R_{1}$ is independent of $t_{0}, s_{1}$ and $t_{1}$ while $T_{1}$ is independent of $s_{p}$. This states that the nearly circular cylinder does not experience any reflection (up to first order) if it has the shape $r=a\{1+\epsilon c(\theta)\}$, where

$$
c(\theta)=\frac{t_{0}}{2}+s_{1} \sin \theta+t_{1} \cos \theta .
$$

Again $T_{1}$ is independent of the $s_{p}$ 's so that if the shape function $c(\theta)$ is of the form $c(\theta)=\sum_{p=1}^{\infty} s_{p} \sin p \theta$, then $T_{1}$ vanishes. These observations give some interesting physical results discussed below.

\section{Discussion}

Taking $\epsilon=0.001$ and 0.0001 we have computed $|T|=\left|T_{0}+\epsilon T_{1}\right|$ for the cylinders

$$
r=a\{1+\epsilon c(\theta)\}
$$

with

$$
c(\theta)=A \sin \theta+B \cos \theta,
$$

for $B=0.1$ ( $A$ being arbitrary), $a / f=0.5$ and for various values of $K a$ between 0.1 and 1.7. A set of representative values is given in Tables 1 and 2.

It is observed from (3.19) that for a sequence of cylinders with cross-sections given in (4.1), $R_{1}=0$, that is, the reflection coefficient up to first order of $\epsilon$, vanishes. This implies that $|T|=\left|T_{0}+\epsilon T_{1}\right|$ is nearly equal to unity. This is also reflected in the numerical results for $|T|$ in Tables 1 and 2. 
TABLE $1 . \epsilon=0.001, a / f=0.5$.

\begin{tabular}{ccc}
\hline$K a$ & $\left|T_{0}\right|$ & $|T|$ \\
\hline 0.1 & 1.000012 & 1.000012 \\
0.3 & 1.000549 & 1.000549 \\
0.5 & 1.000775 & 1.000775 \\
0.7 & 1.000421 & 1.000421 \\
1.0 & 1.000077 & 1.000080 \\
1.3 & 1.000009 & 1.000065 \\
1.5 & 1.000002 & 1.000229 \\
1.7 & 1.000000 & 1.000800 \\
\hline
\end{tabular}

TABLE 2. $\epsilon=0.0001, a / f=0.5$.

\begin{tabular}{ccc}
\hline$K a$ & $\left|T_{0}\right|$ & $|T|$ \\
\hline 0.1 & 1.000012 & 1.000012 \\
0.3 & 1.000549 & 1.000549 \\
0.5 & 1.000775 & 1.000775 \\
0.7 & 1.000421 & 1.000421 \\
1.0 & 1.000077 & 1.000076 \\
1.3 & 1.000009 & 1.000009 \\
1.5 & 1.000002 & 1.000003 \\
1.7 & 1.000000 & 1.000008 \\
\hline
\end{tabular}

Also, it is observed from (3.20) that $|T|$ for these cylinders is independent of $A$. More generally if $c(\theta)=\sum_{p=1}^{\infty} s_{p} \sin p \theta$, then from (3.19) and (3.20) it is found that $R_{1}=0, T_{1}=0$ (of course $R_{0}=0$ ). This implies that for the nearly circular cylinders with the shape function $c(\theta)$ mentioned above, the transmissivity is totally unaffected by its noncircularity.

Again from Table 1, it is found that for $\epsilon=0.001,|T|$ does not differ from $\left|T_{0}\right|$ (up to six decimal places) for $K a<0.9$ where $T_{0}$ is the transmission coefficient for the circular cylinder $r=a$. Again $|T|$ differs from $\left|T_{0}\right|$ beyond the fourth decimal place for $K a$ lying between 1.0 and 1.3. However for $K a \leq 1.4$ the said difference occurs at the fourth decimal place.

Also from Table 2, it is found that for $\epsilon=0.0001,|T|$ does not differ from $\left|T_{0}\right|$ (up to six decimal places) for $K a \leq 0.9$, but for $K a \geq 1.0$, this difference occurs beyond the fifth decimal places. This shows that the effect of $\epsilon$ on $|T|$ is not of much significance for various values of the parameter $K a$ and $K f$. Thus for a sequence of nearly circular cylinders whose cross-sections are given in (4.1), the reflection coefficient up to first order vanishes and the transmission coefficient up to first order does not differ significantly from that of the circular cylinder. It may also be observed that if a nearly circular cylinder $r=a\{1+\epsilon c(\theta)\}$ has sufficient continuity, then the Fourier coefficients $s_{j}$ and $t_{j}$ in (3.18) will decay rapidly with $j$ and a three-term series as given in (3.21) is a good approximation to many fairly smooth cylinders. This implies that for many smooth nearly circular cylinders, the result that for the circular cylinder the reflection coefficient vanishes, is approximately valid.

\section{Appendix: Derivation of the condition (3.1)}

The condition (2.3) is

$$
\frac{\partial \phi}{\partial n}=0 \quad \text { on } r=a\{1+\epsilon c(\theta)\}, \quad 0 \leq \theta \leq 2 \pi .
$$


Let $\alpha$ be the angle between the radius vector and a tangent to the curve $r=r(\theta)$ at $(r, \theta)$, then

$$
\frac{\partial \phi}{\partial n}=\frac{\partial \phi}{\partial r} \sin \alpha-\frac{1}{r} \frac{\partial \phi}{\partial \theta} \cos \alpha
$$

Noting that $\cot \alpha=r^{\prime}(\theta) / r$ on $r=a\{1+\epsilon c(\theta)\}$, we find $\sin \alpha=1+\mathrm{O}\left(\epsilon^{2}\right)$ and $\cos \alpha=\epsilon c^{\prime}(\theta)+\mathrm{O}\left(\epsilon^{2}\right)$ so that

$$
\frac{\partial \phi}{\partial n}=\frac{\partial \phi}{\partial r}-\frac{\epsilon}{a} c^{\prime}(\theta) \frac{\partial \phi}{\partial \theta}+\mathrm{O}\left(\epsilon^{2}\right) \quad \text { on } r=a\{1+\epsilon c(\theta)\} .
$$

Expanding $\partial \phi / \partial r(r, \theta), \partial \phi / \partial \theta(r, \theta)$ on $r=a\{1+\epsilon c(\theta)\}$ about $r=a$ by Taylor series expansion and using the harmonic property of $\phi$, we find from (A.2) that condition (A.1) is approximately equivalent to

$$
\frac{\partial \phi}{\partial r}-\epsilon\left[c(\theta) \frac{\partial \phi}{\partial r}+\frac{1}{a} \frac{d}{d \theta}\left\{c(\theta) \frac{\partial \phi}{\partial \theta}\right\}\right]+\mathrm{O}\left(\epsilon^{2}\right)=0 \quad \text { on } r=a .
$$

\section{Acknowledgement}

The authors take this opportunity to thank the referees and the associate editor for their comments and suggestions in revising the paper in the present form. SB also thanks the National Board For Higher Mathematics for the award of a Post-Doctoral Fellowship.

\section{References}

[1] W. R. Dean, "On the reflection of a surface waves by a submerged cylinder", Proc. Camb. Phil. Soc. 44 (1948) 483-491.

[2] D. V. Evans, "A note on waves produced by the small oscillations of a partially immersed vertical plate", J. Inst. Math. Applics 17 (1976) 135-140.

[3] Z. S. Gradshteyn and I. M. Ryzhik, Tables of integrals, series and products (Academic Press, 1980).

[4] H. Levine, "Scattering of surface waves by a submerged cylinder", J. Math. Phys. 6 (1965) 12311243.

[5] B. N. Mandal and S. Banerjea, "A note on waves due to rolling of a partially immersed nearly vertical plate", SIAM J. Appl. Math. 51 (1991) 930-939.

[6] B. N. Mandal and A. Chakrabarti, "A note on diffraction of water waves by a nearly vertical barrier", IMA J. Appl. Math. 43 (1989) 157-165.

[7] B. N. Mandal and P. K. Kundu, "Ascattering of water waves by a submerged nearly vertical plate", SIAM J. Appl. Math. 50 (1990) 1221-1231.

[8] T. P. Ogilvie, "First and second order forces on a cylinder submerged under a free surface", J. Fluid Mech. 16 (1963) 451-472.

[9] F. Ursell, "Surface waves on deep water in presence of submerged circular cylinder", Proc. Camb. Phil. Soc. 46 (1950) 141-155. 